\title{
Application game and low-cost BCI measurement of electrical scalp activity as an additional tool to test children with ADHD
}

\author{
Almudena Serrano-Barroso ${ }^{1}$, Roma Siugzdaite ${ }^{2}$, Caroline G. Forlim ${ }^{3,4}$, Jaime \\ Guerrero-Cubero ${ }^{5}$, Alberto J. Molina-Cantero ${ }^{5}$, Isabel M. \\ Gómez-González ${ }^{5}$, Elena Nú̃ez-Castellar³, Daniele Marinazzo ${ }^{3}$, Juan C. \\ López ${ }^{1}$, Juan P. Vargas ${ }^{1}$ \\ ${ }^{1}$ Dpto. de Psicologá Experimental. Universidad de Sevilla \\ ${ }^{2}$ Dept. of Experimental Psychology. Faculty of Psychology and Educational Sciences. \\ University of Ghent \\ ${ }^{3}$ Dept. of Data Analysis. Faculty of Psychology and Educational Sciences. University of \\ Ghent \\ ${ }^{4}$ University Clinic Hamburg-Eppendorf, Clinic and Policlinic for Psychiatry and \\ Psychotherapy, Hamburg, Germany \\ ${ }^{5}$ Dpto. de Tecnología Electrónica. ETS Ingeniería Informática. Universidad de Sevilla
}

\section{Corresponding author}

\author{
Almudena Serrano-Barroso \\ Dpto. de Psicología Experimental. \\ Universidad de Sevilla \\ Camilo José Cela, s/n \\ 41018 Sevilla. SPAIN \\ Tel: +34 954550119 \\ Email: aserrano3@us.es
}




\begin{abstract}
Objective: The aim of the present study is to identify attentional biomarkers by using BCI technology of the Attention Deficit Hyperactivity Disorder (ADHD) associated with several pejorative outcomes that are difficult to detect using only behavioral testing. We investigated whether Attention measures using a BCI application game in combination with a neuropsychological test can help to discriminate between participants attending to developmental differences on attentional skills.
\end{abstract}

Materials and Methods: The GokEvolution application game was designed to train and improve attention and to provide an additional measure to identify attentional problems in children. Attention and Meditation changes registered with NeuroSky Mindwave in combination with the CARAS-R psychological test were used to identify attentional skills and impulsivity in 52 non-ADHD and 23 ADHD children from 6 to 12 years old.

Results: The analyses revealed the usefulness of GokEvolution, when considering the measure of Attention. The ADHD group showed lower levels of Attention and more variability in brain responses when compared to the control group. In the ADHD group we found a strong significant negative correlation between attention at the last level of the game. Likewise, a negative correlation with impulsivity was found.

Conclusion: Our results suggest that the system was able to map the low attention profile of the ADHD group when compared to control group and that it holds potential to be used in clinical settings as a screening tool previous to diagnostic.

Keywords: Brain Computer Interface (BCI), diagnostic tools, ADHD, EEG, Neurofeedback, NeuroSky Mindwave.

\title{
Introduction
}

ADHD is a multidimensional disorder, characterized by a mixed pattern of symptoms that can be divided into two categories: 1) lack of attention and 2) hyperactivity plus impulsiveness. The latter includes behaviors such as lack of attention to details, excessive talking and excessive motor activity ${ }^{1}$. ADHD children display these characteristics early in development and in excessive ways causing social functioning difficulties. Impulsive behavior is one of the most disabling features. since it has a very negative impact on the social, emotional and cognitive aspects 
their normal development. The prevalence is estimated at about $5 \%$ for children ${ }^{2}$. ADHD includes inattention, hyperactivity and impulsive subtypes that constitute separable but substantially correlated dimensions ${ }^{3}$.

Attention can be defined as the ability to focus continuously on a particular action, thought or object ${ }^{4}$. Several physiological markers can be used to indicate attention levels: the regional cerebral blood flow ( $\mathrm{rCBF}$ ), which increases during attentional tasks like reading, naming, etc. ${ }^{5}$; the eye pupil diameter ${ }^{6}$ or blinking rate $^{7}$ which increases or decreases respectively as attention increases ${ }^{6}$; markers derived from the ElectroEncephaloGraphy (EEG) activity 2,8 ; etc.

Our interest is focused on EEG correlates but the measurement of these signals requires a controlled unnatural lab environment, complex technical requirements and time-consuming preparation of a costly setup. To overcome these issues, new inexpensive devices based on Brain Computer Interface (BCI) are recently being used to perform studies in natural contexts which might likely be of practical value for clinical use in the near future. Complex EEG- BCI systems require several electrodes placed on the scalp ${ }^{9}$ and are not portable, which might be a problem if measures need to be done outside the lab. Furthermore, a traditional EEG has complex technical requirements, costly and time-consuming preparation of the entire setup, so this new generation of BCI devices hold great potential to be used in clinical settings in the near future.

Some companies, such as Emotiv and NeuroSky, have released portable wireless BCI headsets oriented to the consumers. Comparative research between both low-cost systems revealed that NeuroSky is more user-friendly and easier to set up and maintain ${ }^{10}$. These features are advantageous for clinical purposes. The NeuroSky device has the potential to make the evaluation of participants, especially children, more accessible and efficient.

The NeuroSky MindWave (MW) has been developed as a non-invasive tool with a dry electrode located in the left side of the frontal area corresponding to FP1 position. It provides information through a Bluetooth ${ }^{11}$ connection that can be classified in three levels of processing. From the lowest to higher levels, they are: 1) raw EEG signal, 2) power bands (alpha, beta, theta, delta and gamma), and 3) the eSense Brainwave Patterns of Attention and Meditation. Power bands and eSense signals help to reduce the preprocessing of the raw signals in external devices and allow the use of digital systems with low computation resources, minimizing also the costs and time of the analysis in contrast to other technologies as virtual reality $(\mathrm{VR})^{12}$.

Attention and Meditation values are reported on a relative scale, ranging from 1 up to 100. The proprietary algorithms to compute attention and meditation meters are based on both temporal and frequency domains. The exact algorithm has not been published but the manufacturer asserts that the former has more emphasis on beta wave, whereas the latter on the alpha band. Several researchers have included the MW in their studies, showing the feasibility and reliability of using this technology in detecting or measuring emotional states $3,13,14$, Attention ${ }^{15,16,17,18}$ and Meditation states ${ }^{17,19}$. It has been shown that MW provides accurate readings 
regarding attention and a positive correlation between the Attention level reported on this device and the self-reported attention levels of the participants ${ }^{18}$. A similar study concluded that the MW accurately measured the overall level of mental attention ${ }^{20}$.

Due to the lack of handy and attractive tools to evaluate attentional biomarkers in children,researchers have been developing games for training attention based on Neuro feedback ${ }^{21}$ (NF). For instance, in a recent study ${ }^{22}$, two sets of electrodes were used to control the position of an object on a computer screen by means of attention. Results showed that a high percentage of participants $(70 \%)$ in the experiment could control the game using only one electrode which shows the feasibility of detecting attention with a reduced number of electrodes. Another study ${ }^{23}$ showed that there is a significant improvement in children with ADHD when NF is included in treatment. The theta/beta ratio or the slow cortical potentials (SCP) were combined together as control signal for NF. Moreover, two games were developed with the same NF control signals ${ }^{24}$. In one of them, a boy on a rope moves ahead if theta activity is reduced and the beta activity increased simultaneously for a period of time. In the other game, a ball moves upwards or downwards according to the SCP. With each correct trial, a part of the covert picture becomes visible. Results showed that theta/beta training decreased the posterior-midline theta activity whereas the SCP training increased the central-midline alpha activity. Both facts are associated with improvements in the German ADHD rating scale. Using theta/beta ratio and electrical muscular activity (EMG) as control signals in a game $^{25}$ where children had to keep a ball over a cone, a smiley smiling or a monkey climbing a palm tree. The training benefits ADHD children by improving their attention and reaction times. In all these studies, the number of sessions to make the NF treatment effective is high. Here, we use the NF application game and the BCI technology to obtain markers for ADHD with a low number of sessions, minimizing the influence of the NF on the marker to measure.

BCI technologies represent new horizons for science and technology providing feedback to users by enabling skills training and improvement ${ }^{18}$. In addition, it offers the possibility of registering certain clinical populations in a dynamic manner. BCI could be used in diagnostics of mental disorders to evaluate paradigms, to identify biological markers of impulsivity and to define specific endophenotypes. Moreover, the combination of portable devices and the development of clinical applications could offer a new perspective for clinicians and researchers.

With this aim, we designed a free to download application called GokEvolution App for the evaluation of attentional biomarkers using a game in order to attract the attention of children and to encourage good performance on the test.

Several studies have reported significant variations in the theta, alpha and beta bands in the EEG recordings for several types of ADHD patients ${ }^{26,27}$. Therefore, it might exist a relationship between the Meditation and Attention indexes of MW and 
ADHD. In this study, we will use Attention to analyze the existence of relationships between them and the ADHD groups. We expect that, the combination of the Attention measure recorded by the NeuroSky and the scores of a neuropsychological battery of tests can help to discriminate between participants showing developmental differences on attentional skills.

In this paper, we investigated sustained attention measured through the analysis of the EEG index provided by the NeuroSky NeuroSky device while participants played a game using the GokEvolution Application. We compared the profiles of ADHD children and controls.

\section{Materials Methods}

\subsection{Participants}

52 controls ( 32 boys, 20 girls, mean age 8.98 years, std 1.25) and 23 ADHD (18 boys, 5 girls, mean age 9.5 years, std 1.55) children were evaluated. All the participants had normal or corrected normal vision and no history of neurological disorders. The study was approved by the Comité Coordinador de Ética de la Investigación Biomédica de Andalucía, Junta de Andalucía (Spain) with the code (1221-N-17). Participation in the study was voluntary and participants gave informed consent to the experimental procedure. A convenience random recruiting of control subjects was done for this study. ADHD was the only clinical condition with which they were diagnosed. None of the children with ADHD was on medication during the experiment. Children taking methylphenidate (MPH) had been off medication for 24 hours. No medication other than MPH was used.

\subsection{Clinical Instruments}

Test on Perception of Differences CARAS-R ${ }^{28}$ was used to identify attentional skills as sustained and selective attention and impulsivity behavior in children from 6 to 12 years old. The main task of the test is to identify differences between similar elements. We used it to analyze two measures of our interest: 1) effectiveness related to attention, and 2) impulsivity. Effectiveness in attention (AE) is based on the number of correct answers (A) and errors (E) made during the test. AE index evaluates the performance during the test, penalized by the number of errors, which is subtracted from the number of correct answers. Impulsivity measurement is defined as the index of impulsivity ICI that indicates the proportion of effective and total performance; and is calculated by using this formula:

$$
I C I=\frac{A-E}{A+E} \cdot 100 \%
$$

The combination of the effectiveness and impulsivity variables expressed in 
enneatypes allowed us to classify the children into two groups, the effective and nonimpulsive group and the effective and impulsive group.

\subsection{Design of GokEvolution Application Game}

An attractive and colorful design was chosen to build this application. We used the evolutions of the character (different hair color and shape) as markers of the level of the game. The game was codified under an Android format that could be downloaded on devices as mobile phones or tablets and had simple instructions and a customizable level of difficulty.

\subsection{Training protocol of GokEvolution}

The game had five difficulty levels increasing from level 0 to level 4 wherein the character appears with a different hair color and shape, representing his evolution. The aim of the game is to achieve the complete evolution of a character through five difficulty levels. During the five minutes the game lasts, the left frontal brain activity is recorded using MW, which also sends the Attention and Meditation meters at a rate of $1 \mathrm{~Hz}$. The Attention level demanded by the game increases as the difficulty level does.

In each level, the character shows his evolution with different hair color and shape. To progress in the game, players must gain points by staying in an attentive state; otherwise, they could lose points. The game considers that the player is in an attentive or inattentive state when the attention meter sent by MW is ranged from 50 up to 100 , or from 0 up to 50 respectively. According to the difficulty level, the number of points added/subtracted to/from a global score changes. Table 1 summarizes the quantities used in each level. Please note that, as the difficulty level increases, a lesser quantity of points are added in attentive state and a greater number of them are subtracted when inattentive. To go on the following levels the player must accumulate a certain number of points that also changes according to the level. A player, who could always stay in an attentive state during the game, would finish the five levels in $5 \mathrm{~s}, 9 \mathrm{~s}, 14 \mathrm{~s}, 20 \mathrm{~s}$ and 70 s respectively. This means that it would take approximately $2 \mathrm{~min}$ to complete the evolution of the character with a perfect performance.

Importantly, players could monitor their performance in real time (Neurofeedback) through the main screen of the game that shows two horizontal bars indicating the level of Attention (MW meter) and the accumulated points gained in each level (Table 1). Both bars were scaled in a range between 0 and 100. Once the game was over, we obtained the average of Attention and Meditation per level (per subject).

Table 1. Values added/subtracted to global score in each level of the game according to the level of attention. 


\begin{tabular}{c|c|c|c|c|c}
\multicolumn{9}{c}{ Level } \\
\multicolumn{1}{c}{} & 0 & 1 & 2 & 3 & 4 \\
\hline Attentive & +18 & +16 & +14 & +12 & +10 \\
\hline Inattentive & -2 & -3 & -4 & -5 & -6 \\
\hline
\end{tabular}

\subsection{Data analysis}

Non-parametric Kolmogorov-Smirnov test was used to analyze differences between control and ADHD groups per level. Significance level was set to 0.05 corrected for the multiple comparison (divided by number of levels that were considered $(0.05 / 5=0.01))$ and Bonferroni correction for multiple tests. The levels were set from 0 to 4 , nonetheless we excluded level 4 from the analysis because many children did not complete it. As a final step, we correlated the NeuroSky indexes of Attention and Meditation with the behavioral measures (effectiveness and impulsivity from CARAS-R test).

\section{Results}

\subsection{GokEvolution App. game and NeuroSky}

We developed the GokEvolution BCI application game to test children with ADHD. In the game, the attention measured by NeuroSky was monitored online and this information was used to complete the levels.

According to the design of the game children were able to modulate their brain activity using the Neurofeedback Thus, we expected subjects with high attentional level to complete a level faster. In addition we expected higher attentional levels and more time to complete in the last levels. To test our BCI application game in the control group, we plotted attention average and the total time to complete a level per subject (Figure 1). Results agreed as the expectations described above.

To check for possible attentional differences between the control group and ADHD one,we calculated the Attention distribution across all levels (Figure 2). ADHD group showed lower and more variable average Attention than the control one, across all levels. The difference between groups was significant in the first $(p=0.0397, d=0.56)$ and the third level $(p=0.018, d=0.63)$. Level zero $(p=0.075)$ and the second $(p=0.231)$ levels did not show group differences. We excluded the last level (level 4) from the analysis because many children did not complete it.

NeuroSky provided additional parameter to Attention, called Meditation, nonetheless, Meditation was not used in our BCI application game. Therefore, we expected no variation of the Meditation level between groups and across levels. In this way, meditation was used as a control measure and the same statistical analysis described above was done. As expected, no significant differences between groups across levels were found. 
From the behavioral point of view, inability to finish the game could be related to the lack of Attention paid to the game, since to finish the game the subjects had to maintain high levels of Attention during all levels. This is specially applied to the completion of level 4, the last one. Therefore, each group, control and ADHD, was divided into two subgroups: participants that completed all five levels of the game and the participants that did not complete the last level of the game.

Figure 1. Mean Attention values versus completion time for each level. As expected, these two variables follow an inverse relationship in each level.

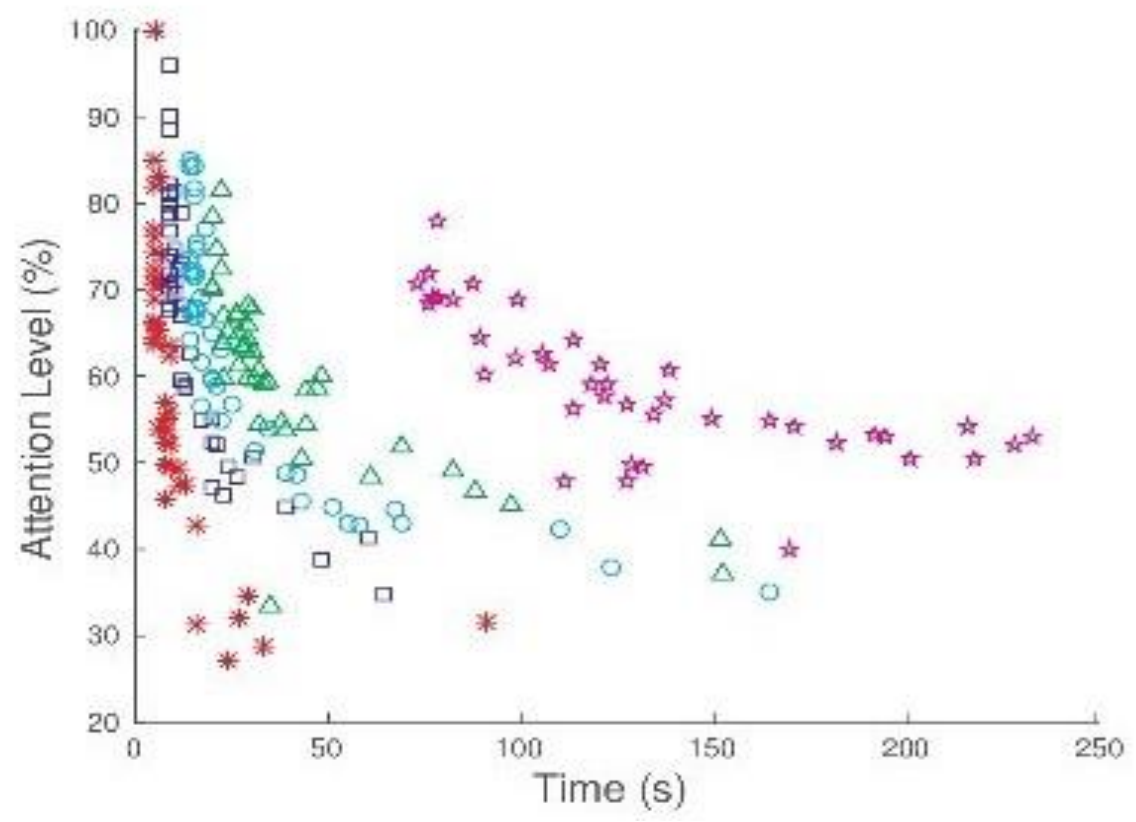

Figure 2. Comparison of mean Attention values between ADHD and control groups in each game level.

ADHD

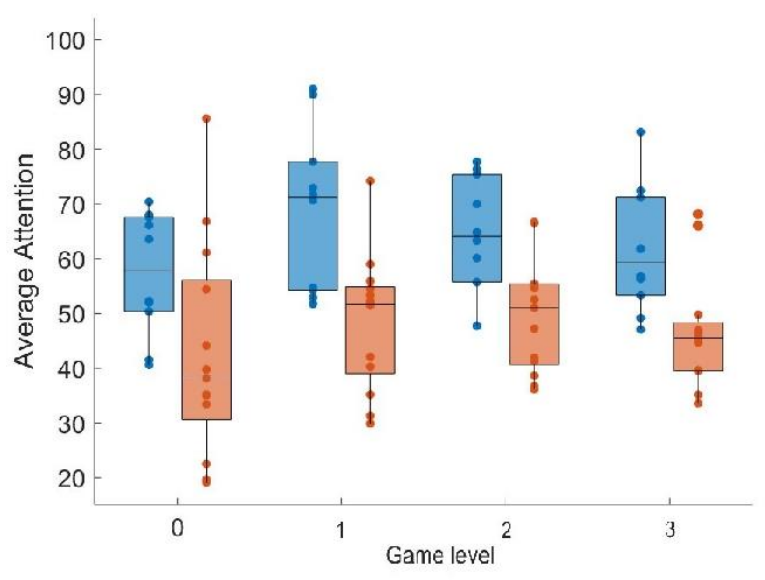

Control group

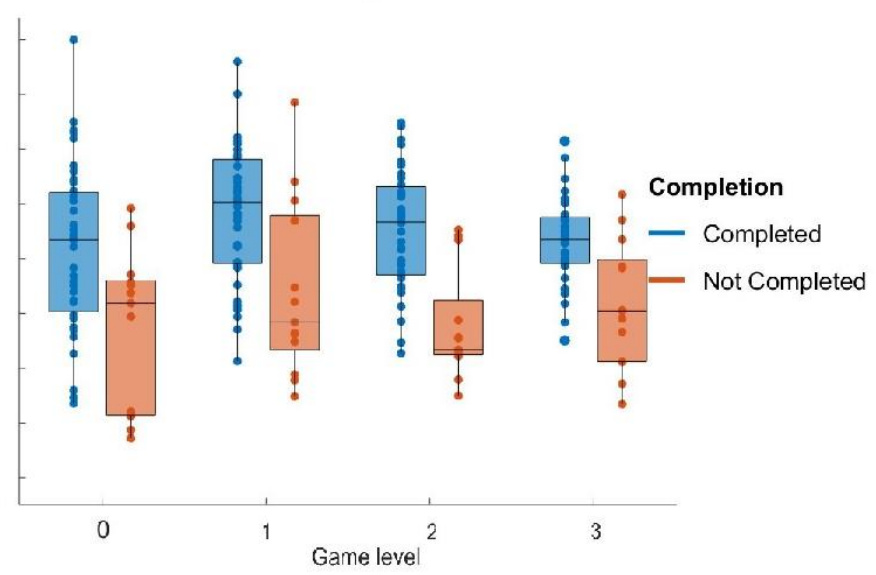


We compared the output of the Attentional level from NeuroSky between these subgroups (Figure 3) and found that there was a significant difference in Attention between them. We observed significant higher Attention in the group that completed all levels, both in control group and ADHD group. When analyzing only the ADHD group, significant statistical differences were found in level $1(p=0.009, d=1.09)$ and the level $2(p=0.0017, d=1.64)$. When analyzing the control group, significant statistical differences were found in the level $0(p=0.0016, d=1.49)$, level 3 $(p=0.00005, d=1.47)$ and $4(p=0.0007, d=1.36)$. Showing a trend on the 0 level in ADHD ( $\mathrm{p}=0.0148, \mathrm{~d}=0.86)$ and control $(\mathrm{p}=0.036, \mathrm{~d}=1.02)$ groups.

Figure 3. Comparison of mean Attention values between groups which completed or did not the five levels of the game.

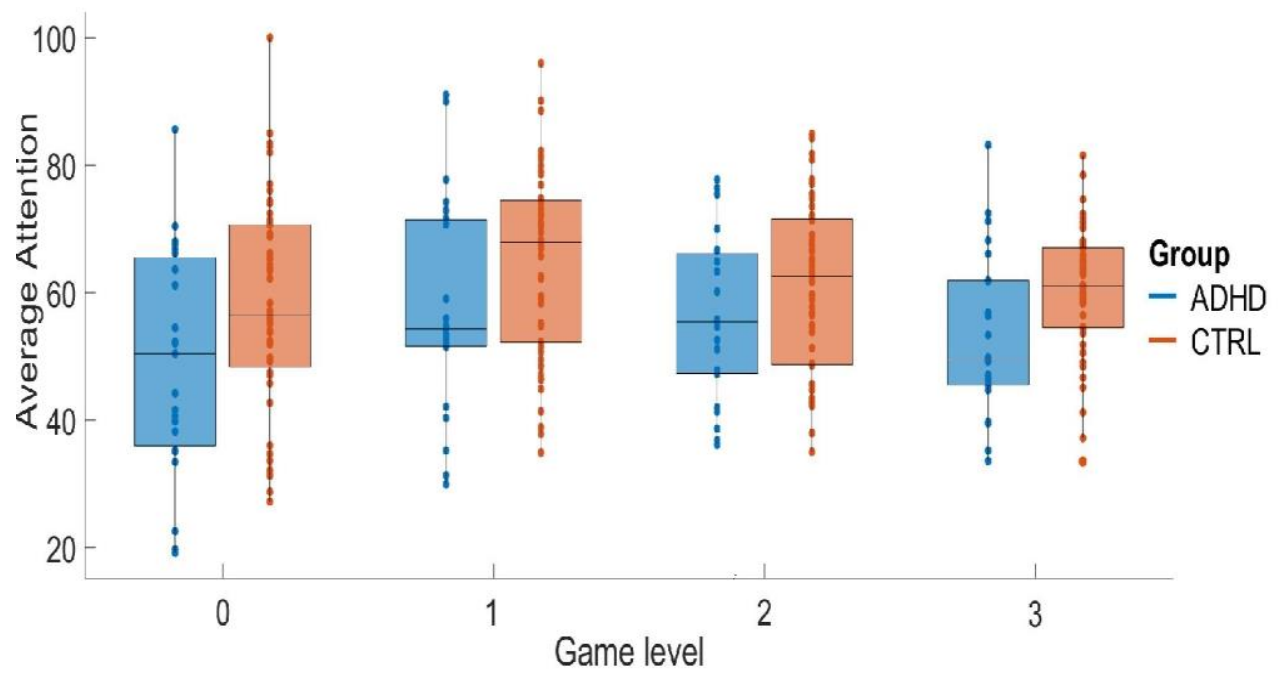

The same statistical analysis was done for meditation parameter from NeuroSky and no significant difference in Meditation were found (Figure 4). No significant differences between the groups: ADHD completed vs. non completed $(\mathrm{p}=0.4488 ; \mathrm{p}=0.5405 ; \mathrm{p}=0.8279 ; \mathrm{p}=0.2270$ ), Controls completed vs. non completed $(\mathrm{p}=0.4488 ; \mathrm{p}=0.5405 ; \mathrm{p}=0.8279 ; \mathrm{p}=0.2270)$. The comparison between groups that completed or not the game showed also no significant differences between ADHD and control group. 
Figure 4. Comparison of mean Meditation meter between groups that completed or did not complete the game.

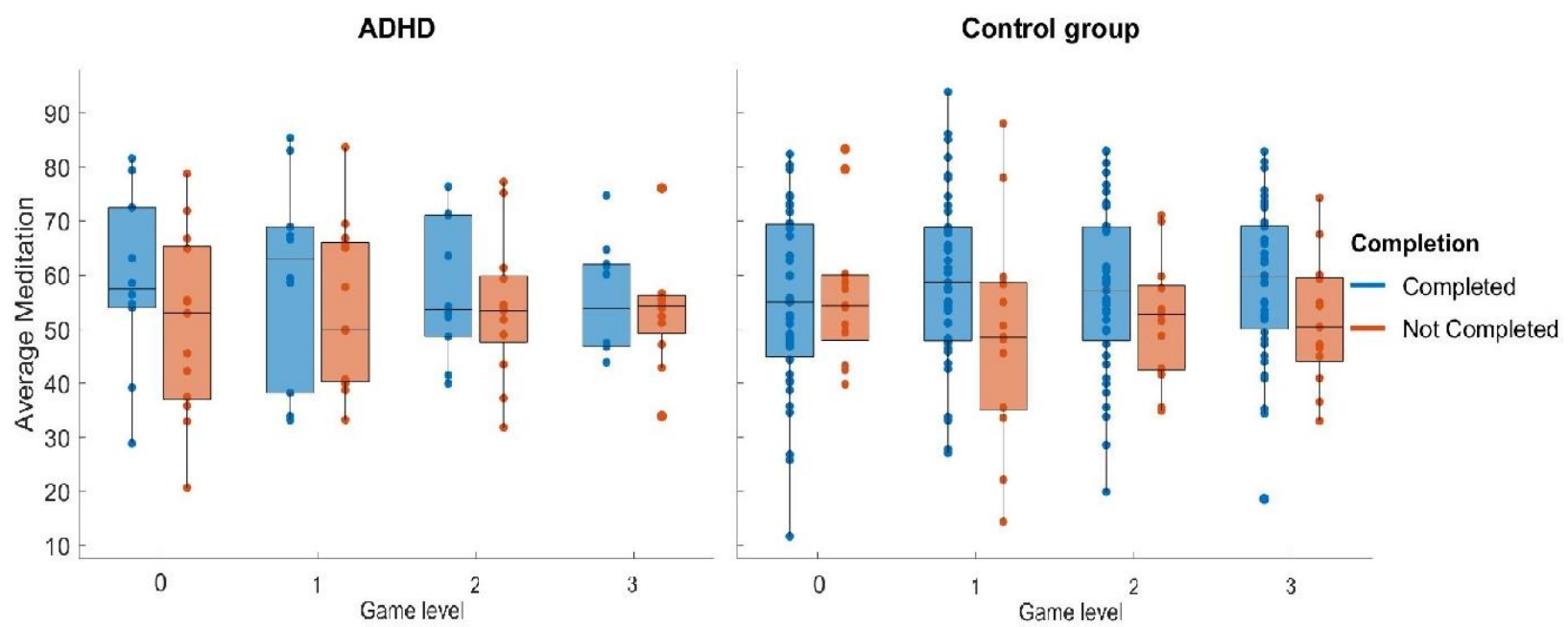

\subsection{Clinical measurements}

The clinical measurements, effectiveness (effective and ineffective) and impulsivity (impulsive and non-impulsive), were obtained from CARAS-R test. We investigated whether effectiveness and impulsivity were associated with the completion of all levels. The subgroups were the same as in section 2.1.

There was a high variability in the scores on effectiveness and impulsivity in ADHD group, especially because after the subdivision the groups were marginally reduced. The descriptive analysis about effectiveness and impulsivity of this subdivision is presented in the Tables 2 and 3.

According to the rules to complete the game and based on effectiveness of the CARASR test: for ADHD group the effective criterion at the test indicates that they were effective but were not able to complete the game. Same for control incomplete group, according to the test 13 were effective but were not able to complete the game. Thus, NeuroSky had more accuracy regarding the attentional biomarker. Besides this, we could say that NeuroSky was measuring Attention well but according to the test we found 8 inattentive controls although they pass the game. Therefore, CARAS-R was not good enough to determine attention.

Table 2. Subdivision of ADHD and controls in completed and incomplete on effectiveness and impulsivity. 


\begin{tabular}{c|c|c|c|c|c} 
& Effective & Ineffective & Impulsive & Nonimpulsive & Total \\
\hline ADHD completed & 3 & 7 & 9 & 1 & $\mathbf{1 0}$ \\
\hline ADHD incompleted & 8 & 5 & 7 & 6 & $\mathbf{1 3}$ \\
\hline Total & $\mathbf{1 1}$ & $\mathbf{1 2}$ & $\mathbf{1 6}$ & $\mathbf{7}$ & $\mathbf{2 3}$ \\
\hline Controls completed & 38 & 0 & 9 & 29 & $\mathbf{3 8}$ \\
\hline Controls incompleted & 13 & 1 & 4 & 10 & $\mathbf{1 4}$ \\
\hline Total & $\mathbf{5 1}$ & $\mathbf{1}$ & $\mathbf{1 3}$ & $\mathbf{3 9}$ & $\mathbf{5 2}$ \\
\hline
\end{tabular}

Table 3. Subdivision of ADHD and controls in completed and incomplete on performance.

\begin{tabular}{c|c|c|c|c|c} 
& Effective & Ineffective & Impulsive & Nonimpulsive & Total \\
\hline ADHD completed & 0 & 1 & 8 & 1 & $\mathbf{1 0}$ \\
\hline ADHD incompleted & 4 & 2 & 6 & 1 & $\mathbf{1 3}$ \\
\hline Total & $\mathbf{4}$ & $\mathbf{3}$ & $\mathbf{1 4}$ & $\mathbf{2}$ & $\mathbf{2 3}$ \\
\hline Controls completed & 38 & 0 & 9 & 29 & $\mathbf{3 8}$ \\
\hline Controls incompleted & 13 & 1 & 4 & 10 & $\mathbf{1 4}$ \\
\hline Total & $\mathbf{5 1}$ & $\mathbf{1}$ & $\mathbf{1 3}$ & $\mathbf{3 9}$ & $\mathbf{5 2}$ \\
\hline
\end{tabular}

\subsection{Correlations of Neuroactivation and Clinical Measures}

We correlated Attentional levels with clinical measures of effectiveness and impulsivity to investigate the relationship between behavioral and the brain activation measures (Figure 5). In the ADHD group that completed all levels of the game, we found a significative negative correlation between level 3 of the GokEvolution game and ICI $(r=-0.68, p=0.0285)$. We did not find any significant correlations in the ADHD group that did not finish the all levels of the game. Level 0 , correlated with effectiveness $(r=-0.54, p=0.045)$. We did not find significant correlations in controls that completed all levels.

Figure 5. Correlations between Attention and Clinical Measures of effectiveness and impulsivity in groups which completed or did not the five levels of the game. 

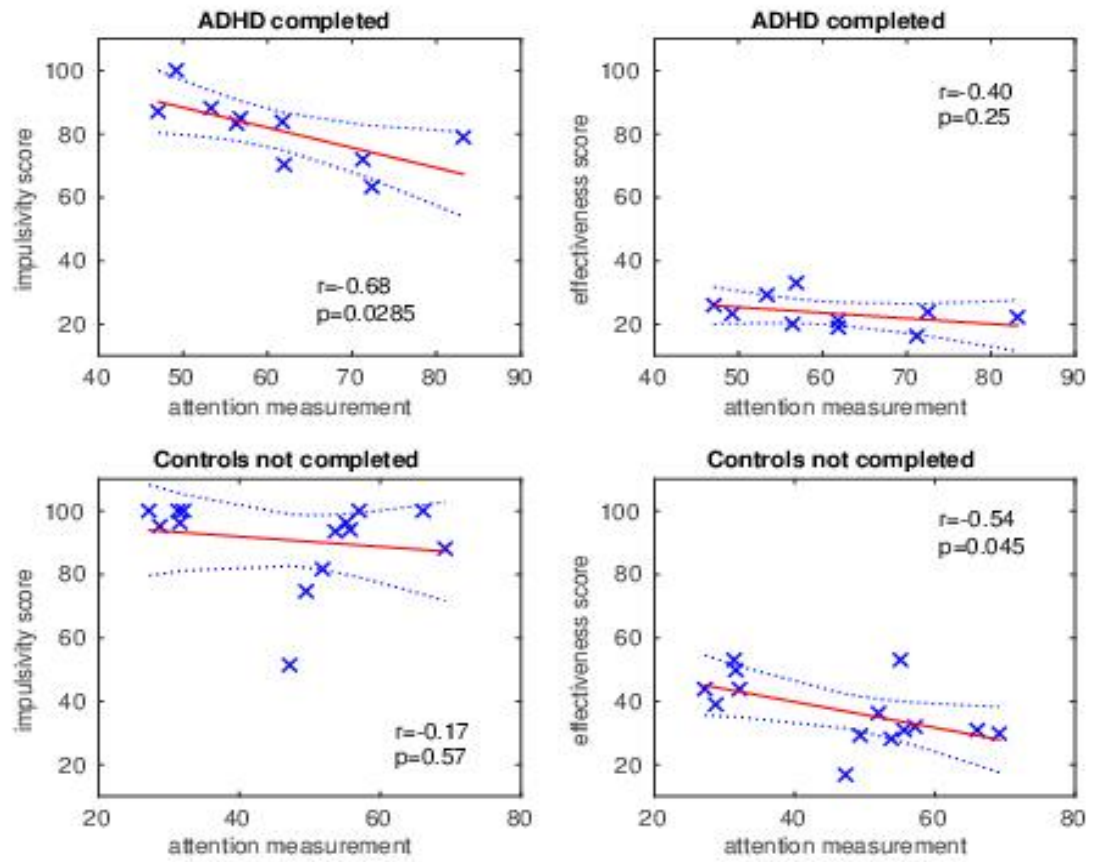

\section{Discussion}

We developed a BCI application game in which the Attention level measured by NeuroSky was monitored and used to complete different levels of difficulty.

NeuroSky proved to be sensitive to attentional changes while children played the GokEvolution game. Our preliminary results showed that Attention variability given by the $\mathrm{BCI}$ device can be used as an attentional biomarker. We found higher Attentional levels in the control group but not in the ADHD group that would support the definition about the first category of impairment a lack of attention in children with ADHD. The crucial levels for these differences were level 1 and 3. Level 1 is the level in which children showed the highest involvement of attention in both groups, though it was significantly higher in controls than in the ADHD group. The differences in the last level of the game, level 4 can be explained by the difficulty to keep concentration for a longer time.

Interestingly, several participants did not finish the game in both groups. Controls who completed all levels should be a clean sample of typically developing children. The subgroup of controls who did not complete the last level, we speculate that it might have some problems in controlling their attention. Furthermore, it could be similar to the ADHD group that did not complete the game, becoming the problematic group that was easy to detect also with the clinical testing. However, the group of ADHDs that finished the game appears to be the group on the border between controls and ADHDs, suggesting the difficulty of their diagnosis. This is the 
first indication to inattentiveness and perhaps, also a way to make a clearer separation within group.

Regarding to the feasibility of the application when considering this subdivision, we also looked at bio-signal measures and we found that the Attentional level of the group that completed the game was significantly higher than that of the group that did not finish it. This behavior was seen in almost all levels of the game. It could be explained by differences in sustained attention between groups over time. Better performance and completion of the game indicates better control of attentional resources.

Finally, we correlated NeuroSky output measurements of Attention and two clinical indicators on effectiveness and impulsivity. We found that in atypical controls (game incompleted) Attention was negatively correlated with effectiveness already at level 0 of the game. That would mean that this group outperform typically at the initial levels of the game but then cannot maintain Attention until the final levels of the game. In addition, in a group of atypical ADHD in the end of the game attention negatively correlated with impulsivity. This could be explained by the idea that high impulsivity individuals may be less prone to attentional difficulties when cognitive load is relatively low. However, when attention switching is involved, high impulsivity is associated with greater difficulty in inhibiting responses and resolving cognitive conflict ${ }^{29}$.

The fact that GokEvolution was developed to be used on a tablet device vastly increases usability of the training program. Due to the easiness to use this application, it takes in total 15 min maximum to prepare the entire setup and to do testing. Thus, it is easy to perform and fun for children. Another major advantage is the fact that the training can also be carried out independently at hospitals, private centers or schools. The application can be used everywhere; there is no need for an internet connection during training. In the future, the validation of the test could be adjusted for age range and accuracy using the levels of difficulty.

The device in combination with the app could work as a prescreening diagnosis tool to detect attentional levels in order to decide faster for targeted tests and to evaluate endophenotypes, which could further our understanding of different behavior disorders related to the attention and impulse control. Recent studies have shown that Autoshaping procedure where a conditioned stimulus (CS) is associated to reward of a food pellet unconditioned stimulus (US), in rats and its application in uman models could help to identify biological markers of impulsivity ${ }^{30,31}$ and define specific endophenotypes. The development of endophenotypes would be more adequate for the effective application of pharmacological and behavioral treatments than traditional classification of mental diseases based on diagnosis scales.

The results observed in the present study are based on the measurements from one electrode (FP1) that is more limited that a multichannel EEG device. Even if we 
managed to show that the device is measuring Attention, in an ideal situation we would expect the better coverage of the brain.

BCI preliminary results showed that Attention variability, given by the BCI device, can be used as an attentional biomarker. We observed that using clinical tests 4 from 13 children with ADHD were defined as showing normal performance, even though they did not complete the level 4 of the game. That diagnosis contradicts the present finding. The question we posed in this study was: can we differentiate correctly these cases using NeuroSky measurements while children play GokEvolution. We were able to detect the variability differences between groups that completed and did not complete the game. Nevertheless, more importantly we were able to distinguish groups that are in the grey area on the border between ADHD and controls (ADHD that completed the game, and controls that did not complete the game).

Taking all together, we hypothesize that by investigating levels of Attention and correlating them with behavioral measures we could characterize our participants into impulsive and non-impulsive as endophenotypes described as Sign Trackers (ST) and Goal Trackers (GT) from the Autoshaping procedure ${ }^{32}$. Studies carried out by our research group have shown that these two groups might follow different patterns regarding impulse control stimuli and the attention span depends on external factors, but with different results. The way this endophenotypes respond to cues may predict vulnerability to compulsive behavioral disorders ${ }^{31}$ and could work as a model to evaluate individual differences regarding impulsivity and attention factors $^{33}$. The analysis at several levels of these endophenotypic differences could further our understanding of different behavior disorders related to the reinforcement system.

\section{Acknowledgments}

This research was supported by PSI2015-65500-P and BES-2013-065007 grants (MINECO, FEDER, UE).

Plots have been made using Gramm by Pierre Morel https://github.com/piermorel/gramm https://doi.org/10.5281/zenodo.59786

Data and code shared in https://figshare.com/s/a471d491e44f6d676cef

\section{Author disclosure statement}

The authors declare no conflict and no competing financial interests.

\section{References}


${ }^{1}$ American Psychiatric Association (2013) Diagnostic and statistical manual of mental disorders (DSM-5®). American Psychiatric Pub.

${ }^{2}$ Polanczyk, G. V., Willcutt, E. G., Salum, G. A., Kieling, C., \& Rohde, L. A. (2014). ADHD prevalence estimates across three decades: An updated systematic review and meta-regression analysis. International Journal of Epidemiology, 43(2), 434442.

${ }^{3}$ Sokolova, E., Groot, P., Claassen, T., Hulzen, K. J., Glennon, J. C., Franke, B., Buitelaar, J. (2016). Statistical Evidence Suggests that Inattention Drives Hyperactivity/Impulsivity in Attention Deficit-Hyperactivity Disorder. Plos One, 11(10).

${ }^{4}$ Castillo MD. La atención. Pirámide. 2009.

${ }^{5}$ Larrue, V., Celsis, P., Bès, A., \& Marc-Vergnes, J. P. (1994). The Functional Anatomy of Attention in Humans: Cerebral Blood Flow Changes Induced by Reading, Naming, and the Stroop Effect. Journal of Cerebral Blood Flow \& Metabolism, 14(6), 958-962.

${ }^{6}$ Iwanaga, K., Saito, S., Shimomura, Y., Harada, H., \& Katsuura, T. (2000). The Effect of Mental Loads on Muscle Tension, Blood Pressure and Blink Rate. Journal of Physiological Anthropology and Applied Human Science, 19(3), 135-141.

${ }^{7}$ Schleicher, R., Galley, N., Briest, S., \& Galley, L. (2008). Blinks and saccades as indicators of fatigue in sleepiness warnings: Looking tired? Ergonomics, 51(7), 982-1010.

${ }^{8}$ Molina-Cantero, A., Guerrero-Cubero, J., Gómez-González, I., Merino-Monge, M., \& Silva-Silva, J. (2017). Characterizing Computer Access Using a One-Channel EEG Wireless Sensor. Sensors, 17(7), 1525.

${ }^{9}$ Nicolas-Alonso, L. F., \& Gomez-Gil, J. (2012). Brain Computer Interfaces, a Review. Sensors, 12(2), 1211-1279.

${ }^{10}$ Das, R., Chatterjee, D., Das, D., Sinharay, A., \& Sinha, A. (2014). Cognitive load measurement - A methodology to compare low cost commercial EEG devices.2014 International Conference on Advances in Computing, Communications and Informatics (ICACCI).

${ }^{11}$ Shirazi, A. S., Hassib, M., Henze, N., Schmidt, A., \& Kunze, K. (2014). Whats on your mind? Proceedings of the 5th Augmented Human International Conference on - $A H 14$.

${ }^{12}$ Díaz-Orueta, U., Garcia-López, C., Crespo-Eguílaz, N., Sánchez-Carpintero, R., Climent, G., \& Narbona, J. (2013). AULA virtual reality test as an attention 
measure: Convergent validity with Conners' Continuous Performance Test. Child Neuropsychology, 20(3), 328-342.

${ }^{13}$ Crowley, K., Sliney, A., Pitt, I., \& Murphy, D. (2010). Evaluating a Brain-Computer Interface to Categorise Human Emotional Response. 2010 10th IEEE International Conference on Advanced Learning Technologies.

${ }^{14}$ Quesada-Tabares, R., Molina-Cantero, A. J., Gómez-González, I., Merino-Monge, M., Castro-García, J. A., \& Cabrera-Cabrera, R. (2017). Emotions Detection based on a Single-electrode EEG Device. Proceedings of the 4th International Conference on Physiological Computing Systems.

${ }^{15}$ Rebolledo-Mendez, G., Dunwell, I., Martvnes-Miron, E. A., Vargas-Cerdan, M. D., de Freitas, S., \& Liarokapis, F. (2009). Assessing the usability of a brain-computer interface (BCI) that detects attention levels in an assessment exercise. 149-158.

${ }^{16}$ Eid, M., \& Fernandez, A. (2013). ReadGoGo!: Towards real-time notification on readers state of attention. 2013 XXIV International Conference on Information, Communication and Automation Technologies (ICAT).

17 Garzotto, F., Gelsomini, M., Pappalardo, A., Sanna, C., Stella, E., \& Zanella, M. (2016). Using Brain Signals in Adaptive Smart Spaces for Disabled Children. Proceedings of the 2016 CHI Conference Extended Abstracts on Human Factors in Computing Systems - CHI EA 16.

${ }^{18} \mathrm{Hammon}$ PS, Sa V. Preprocessing and meta-classification for Brain Computer Interfaces. IEEETransactionson Biomedical Engineering 2007; 54:518-525.

${ }^{19}$ Sosa Jiménez CO, Acosta Mesa HG, Rebolledo-Mendez G, Freitas S. Classification of cognitive states of attention and relaxation using supervised learning algorithms. In Games Innovation Conference (IGIC), 2011 IEEE International 2011; 31-34.

${ }^{20}$ Fong SSM, Tsang WWN, Cheng YTY, Ki WY, Ma AWW, Macfarlane DJ. (2015) Single-channel Electroencephalographic Recording in Children with Developmental Coordination Disorder: Validity and Influence of Eye Blink Artifacts. J Nov Physiotherapy; 5:270.

${ }^{21}$ Heinrich H, Gevensleben H, Strehl U. (2007) Annotation: Neurofeedback-train your brain to train behaviour. J Child Psychol Psychiatry; 48:3-16.

${ }^{22}$ George L, Lotte F, Viciana AR, Lécuyer A. (2011)Using scalp electrical biosignals to control an object by concentration and relaxation tasks: design and evaluation. In Engineering in Medicine and Biology Society, EMBC, 2011 Annual International Conference of the IEEE; 6299-6302.

${ }^{23}$ Gevensleben H, Holl B, Albrecht B, Vogel C, Schlamp D, Kratz O, Studer P, Rothenberger A, Moll GH, Heinrich H. (2009) Is neurofeedback an efficacious 
treatment for ADHD? A randomised controlled clinical trial. J Child Psychol Psychiatry; 50:780-789.

${ }^{24}$ Gevensleben H, Holl B, Albrecht B, Schlamp D, Kratz O, Studer P, Wangler S, Rothenberg A, Moll G.H. Heinrich H. (2009) Distinct EEG effects related to neurofeedback training in children with ADHD: A randomized controlled trial. Int J Psychophysiol; 75:149-157.

${ }^{25}$ Bakhshayesh AR, Hänsch S, Wyschkon A, Rezai MJ, Esser G. (2011) Neurofeedback in ADHD: a single-blind randomized controlled trial. Eur Child Adolesc Psychiatry, 20:481.

${ }^{26}$ Clarke AR, Barry RJ, McCarthy R, Selikowitz M. (1998) EEG analysis in Attention-Deficit/Hyperactivity Disorder: a comparative study of two subtypes. Psychiatry Res; 81:19-29.

${ }^{27}$ Clarke AR, Barry RJ, McCarthy R, Selikowitz M. (2001) Electroencephalogram differences in two subtypes of Attention-Deficit/Hyperactivity Disorder. Psychophysiol; 38:212-221.

${ }^{28}$ Thurstone L.L. Yela M. Percepcion de diferencias (CARAS-R). TEA Ediciones. 2012.

${ }^{29}$ Leshem R. (2016) Relationships between trait impulsivity and cognitive control: the effect of attention switching on response inhibition and conflict resolution. Cogn Process; 17:89-103.

${ }^{30}$ Flagel, S.B., Watson, S.J., Robinson, T.E., \& Akil, H. (2006). Individual differences in the propensity to approach signals vs goals promote different adaptations in the dopamine system of rats. Psychopharmacology, 191, 599-607.

${ }^{31}$ Lopez, J. C., Karlsson, R.-M., \& O’Donnell, P. (2015). Dopamine D2 Modulation of Sign and Goal Tracking in Rats. Neuropsychopharmacology, 40(9), 2096-2102.

${ }^{32}$ Morrow, J. D., Maren, S., \& Robinson, T. E. (2011). Individual Variation in the Propensity to Attribute Incentive Salience to an Appetitive Cue Predicts the Propensity to Attribute Motivational Salience to an Aversive Cue. Behavioural Brain Research, 220(1), 238-243.

${ }^{33}$ Vargas, J. P., Díaz, E., Portavella, M., \& López, J. C. (2016). Animal Models of Maladaptive Traits: Disorders in Sensorimotor Gating and Attentional Quantifiable Responses as Possible Endophenotypes. Frontiers in Psychology, 7, 206. 